BULL. AUSTRAL. MATH. SOC.

VOL. $27(1982), 83-89$.

\title{
FUNCTIONAL EQUATIONS OCCURRING IN THE THEORY OF DELAYED DIFFERENTIAL EQUATIONS
}

\author{
Józef Banaś and StanisLaw WędRychowicz
}

\begin{abstract}
This paper is devoted to duscussion of some functional equations obtained in the theory of delayed differential equations. By means of the Laplace transform distribution solutions of the considered equations are constructed.
\end{abstract}

\section{Introduction}

In this paper we are going to discuss some functional equations which are obtained in connection with considerations on delayed differential equations. The functional equations considered here are not especially complicated and they are special cases of the functional equation of the form $x(t)=F(t, x(\varphi(t)))$, where $x(t)$ is an unknown function. This equation was examined in several papers (cf. [3], [1]). In principle the general aim of the paper is to show how we can obtain functional equations in the theory of delayed differential equations. Moreover, one can show that the knowledge of solutions of those functional equations enables us to find some generalized solutions of considered delayed differential equations. Let us mention that we will consider only analytic solutions of functional equations although they also have nonanalytic solutions which will be shown ( $f f .[3],[7]$ ). The tool which allows us to carry over delayed differential equations to functional equations is the method of Laplace transforms. This method is very often used for solving linear ordinary and partial differential equations (see, for example, [4], [6]).

Received 16 August 1982. 


\section{Delayed differential equation of the first order}

Let us consider the simple delayed differential equation

$$
x^{\prime}(t)=A x(a t), t \geq 0,
$$

with the initial condition

$$
x(0)=x_{0},
$$

(cf. [5]). We shall assume that $A$ and $a$ are real constants and $0<a \leq 1$. It is easy to show that the problem (1)-(2) has the following analytic solution

$$
x(t)=x_{0} \sum_{n=0}^{\infty} \frac{A^{n} a^{(n-1) n / 2}}{n !} t^{n} .
$$

On the other hand we can show (cf. Section 3) that the solution of (1)-(2) is an exponential order, so that we may apply to that problem the Laplace transformation [4] defined by the formula

$$
L[x]=\int_{0}^{\infty} e^{-z t} x(t) d t=f(z),
$$

where $z$ is complex number. Thus we obtain the following functional equation

$$
z f(z)-(A / a) f(z / a)=x_{0}
$$

In the sequel we shall seek an analytic solution of equation (3). First, putting $z=0$, we have $f(0)=-a x_{0} / A$. Further, differentiating both sides of (3) and taking $z=0$ we have $f^{\prime}(0)=-x_{0} a^{3} / A^{2}$. In the same way, by induction we calculate that

$$
f^{(n)}(0)=-x_{0} n ! \frac{a^{(n+1)(n+2) / 2}}{A^{n+1}}
$$

for $n=0,1,2, \ldots$. Now writing the solution of equation (3) as the Taylor series expansion we get

$$
f(z)=-x_{0} \sum_{n=0}^{\infty} \frac{a^{(n+1)(n+2) / 2}}{A^{n+1}} z^{n} .
$$


It is easy to check that the above series converges uniformly on the whole finite complex plane so that the function $f(z)$ is the analytic solution of equation ( 3 ).

Now let us observe that by means of solution (4) we can find a solution of the problem (1)-(2). To do it we have to apply to the series (4) the inverse Laplace transform [6]. Indeed, taking into account that $L\left[\delta^{(n)}\right]=z^{n}, n=0,1, \ldots$, where $\delta(t)$ denotes the Dirac distribution and applying the theorem on convergence of series of generalized functions [6] we obtain

$$
x(t)=-x_{0} \sum_{n=0}^{\infty} \frac{a^{(n+1)(n+2) / 2}}{A^{n+1}} \delta^{(n)}(t),
$$

for $t \geq 0$. The function $x(t)$ given by the above formula is the solution of (1)-(2) in a generalized sense.

It is worthwhile mentioning that we can also obtain a solution of equation (3) in another way. In order to find it we apply the Laplace transform to the analytic solution of (1)-(2) obtained in the beginning. Thus, because of the well-known formula

$$
\int_{0}^{\infty} t^{n} e^{-z t} d t=\frac{n !}{z^{n+1}}, n=0,1,2, \ldots,
$$

we obtain

$$
f(z)=x_{0} \sum_{n=0}^{\infty} \frac{A_{a}^{n}(n-1) n / 2}{z^{n+1}} .
$$

Notice that this series does not converge on the whole finite complex plane and this solution is well defined for all $z \neq 0$. Actually the function $f(z)$ satisfies equation (3) what we can check by a simple calculation.

\section{Another functional equation}

In this section we will consider the delayed differential equation of the second order

$$
x^{\prime \prime}(t)=A x(a t), \quad t \geq 0,
$$

assuming the following initial conditions 


$$
x(0)=x_{1}, x^{\prime}(0)=x_{2} .
$$

Similarly to the previous section we shall assume that $\alpha$ and $A$ are real constants and $0<a \leq 1$.

Let us notice that putting $x_{1}(t)=x(t), x_{2}(t)=x^{\prime}(t)$ we can transform equation (6) to the following system,

$$
\begin{aligned}
& x_{1}^{\prime}(t)=x_{2}(t), \\
& x_{2}^{\prime}(t)=A x_{1}(a t),
\end{aligned}
$$

with the initial condition $\left(x_{1}(0), x_{2}(0)\right)=\left(x_{1}, x_{2}\right)$. The above problem can be rewritten in the following integral form

$$
\left\{\begin{array}{l}
x_{1}(t)=x_{1}+\int_{0}^{t} x_{2}(s) d s, \\
x_{2}(t)=x_{2}+\int_{0}^{t} A x_{1}(a s) d s .
\end{array}\right.
$$

Hence

$$
\left\{\begin{array}{l}
\left|x_{1}(t)\right| \leq\left|x_{1}\right|+\int_{0}^{t}\left|x_{2}(s)\right| d s, \\
\left|x_{2}(t)\right| \leq\left|x_{2}\right|+|A| \int_{0}^{t}\left|x_{1}(a s)\right| d s .
\end{array}\right.
$$

Further, denoting $\varphi_{i}(t)=\max \left[\left|x_{i}(s)\right|: 0 \leq s \leq t\right], i=1,2$, from the last system of the integral inequalities we obtain

$$
\left\{\begin{array}{l}
\varphi_{1}(t) \leq\left|x_{1}\right|+\int_{0}^{t} \varphi_{2}(s) d s, \\
\varphi_{2}(t) \leq\left|x_{2}\right|+|A| \int_{0}^{t} \varphi_{1}(s) d s .
\end{array}\right.
$$

If we denote

$$
\varphi(t)=\left[\begin{array}{l}
\varphi_{1}(t) \\
\varphi_{2}(t)
\end{array}\right], C=\left[\begin{array}{c}
\left.\mid x_{1}\right\rceil \\
\left|x_{2}\right|
\end{array}\right], A=\left[\begin{array}{cc}
0 & 1 \\
|A|, & 0
\end{array}\right],
$$


then, from (8), we have the following inequality

$$
\varphi(t) \leq C+\int_{0}^{t} A_{\varphi}(s) d s .
$$

Hence we get

$$
\|\varphi(t)\| \leq\|C\|+\int_{0}^{t}\|A\|\|\varphi(s)\| d s
$$

and in view of Gronwall's lemma [2] we obtain

$$
\|\varphi(t)\| \leq\|C\| \exp \left(\int_{0}^{t}\|A\| d s\right)=\|C\| \exp (\|A\| t),
$$

where, for example, $\left\|\left(x_{1}, x_{2}\right)\right\|=\max \left[\left|x_{1}\right|,\left|x_{2}\right|\right]$.

Finally we obtain the following inequality

$$
|x(t)| \leq\|C\| \exp (\|A\| t)
$$

which means that a solution of $(5)-(6)$ is of exponential order. This assertion permits us to apply to the problem (5)-(6) the Laplace transformation.

Thus we have

$$
z^{2} f(z)-z x_{1}-x_{2}=(A / a) f(z / a)
$$

Writing the above functional equation in the form

$$
f(z)=a_{1} z^{2} f(a z)-a_{2} z-a_{3}
$$

where $a_{1}=a^{3} / A, a_{2}=a^{2} x_{1} / A, a_{3}=a x_{2} / A$ and looking for an analytic solution of (8) we can - via the standard procedure - obtain

$$
\begin{aligned}
f^{(2 k)}(0) & =-(2 k) ! a^{(k-1) k} a_{1}^{k} a_{3}, \\
f^{(2 k+1)}(0) & =-(2 k+1) ! a^{k^{2}} a_{1}^{k} a_{2},
\end{aligned}
$$

for $k=0,1,2, \ldots$. Thus we have 


$$
f(z)=\sum_{k=0}^{\infty} \frac{f^{(k)}(0)}{k !} z^{k}=-\left(\sum_{k=0}^{\infty} a^{(k-1) k} a_{1}^{k} a_{3} z^{2 k}+\sum_{k=0}^{\infty} a^{k^{2}} a_{1}^{k} a_{2} z^{2 k+1}\right) .
$$

It is easy to check that the above series converges uniformly on the whole funite complex plane so that the function $f(z)$ is an analytic solution of equation ( 8 ).

Now, as in Section 2, applying to the function $f(z)$ the inverse Laplace transform and using the formula $L\left[\delta^{(n)}\right]=z^{n}, n=0,1, \ldots$ we obtain

$$
x(t)=-\left(\sum_{k=0}^{\infty} a^{(k-1) k a_{1}^{k} a_{3} \delta(2 k)}(t)+\sum_{k=0}^{\infty} a^{k^{2}} a_{1}^{k} a_{2} \delta^{(2 k+1)}(t)\right),
$$

for $t \geq 0$. This function is a generalized solution of the problem $(5)-(6)$.

Let us notice that by the same method as in Section 2 we can also obtain a nonanalytic solution of the functional equation (8). Namely it suffices to find an analytic solution of the problem (5)-(6) and then apply the Laplace transformation to it. We omit the details.

Finally, let us observe that a similar method may be used for the more complicated delay differential equation, of an arbitrary order $n$,

$$
\begin{aligned}
x^{(n)}(t) & =A_{1} x^{(n-1)}(t)+A_{2} x^{(n-2)}\left(a_{2} t\right)+\ldots+A_{n} x\left(a_{n} t\right), \\
x^{(i-1)}(0) & =x_{i-1}, i=1,2, \ldots, n,
\end{aligned}
$$

where $A_{i}, a_{i}$ are real constants and $a_{i} \in(0,1)$ for $i=1,2, \ldots, n$.

\section{References}

[1] Józef Banas, Andrzej Hajnosz and Stanis/aw Wędrychowicz, "On existence and asymptotic behavior of solutions of some functional equations", Fronkcial. Ekvac. (to appear).

[2] Philip Hartman, Ordinary differential equations (John Wiley \& Sons, New York, London, Sydney, 1964). 
[3] Marek Kuczma, Functional equations in a single variable (Monografie Matematiyczne, 46. PWN - Polish Scientific Publishers, Warszawa, 1968).

[4] Jan Mikusiński, Rachunek operatorów (Monografie Matematyczne, 30. PWN - Polish Scientific Publishers, Warszawa, 1953).

[5] А.Д. МПиис [A.D. Mүs̆kis], Линеймые дифференииалюне уравнения $c$ запаздива:иим аргументом [Linear differential equations with retarded argument], Second edition (Izdat. "Nauka", Moscow, $1972)$.

[6] Laurent Schwartz; Thêorie des distributions (Publications de I'Institut de Mathématique de I'Université de Strasbourg, IX-X. Hermann, Paris, 1966).

[7] W. Smajdor, "On the existence and uniqueness of analytic solutions of the functional equation $\varphi(z)=h(z, \varphi[f(z)])$ ", Ann. Polon. Math. 19 (1967), 37-45.

Institute of Mathematics and Physics,

Technical University,

35-084 Rzeszów,

Poznańska 1,

Poland. 